\title{
FURTHER PROPERTIES OF THE DYNAMICAL CLOCK A+ INDICATOR IN A TOY MODEL OF PURE DYNAMICAL FRICTION
}

\author{
M. Pasquato ${ }^{1,2}$ \\ Received July 29 2019; accepted October 72019
}

\begin{abstract}
The Alessandrini A+ indicator is a measure of star cluster dynamical evolution based on the mass-segregation of blue straggler stars. A+ is defined as the integral of the cumulative distribution of blue stragglers over log radius, minus a term related to the reference population used. In a companion paper I introduced a model of dynamical friction and calculated the A+ indicator analytically. Here I show further properties of the time evolution of $\mathrm{A}+$, focusing on the physical interpretation of its time derivative $d A^{+} / d t$. I find that $d A^{+} / d t$ is the mean of the reciprocal dyamical friction timescale, weighted by the density of blue stragglers. I show that it is non-negative (as expected based on monotonicity) due to the density of blue-stragglers being non-negative and that, for a radially non-decreasing dynamical friction timescale, $d A^{+} / d t$ is also non-decreasing with time, making $\mathrm{A}+$ a convex function.
\end{abstract}

\section{RESUMEN}

El indicador de Alessandrini A+ es una medida de la evolución dinámica de un cúmulo estelar basada en la segregación por masa de las estrellas rezagadas azules. Se define A+ como la integral de la distribución acumulada de las rezagadas azules sobre el logaritmo del radio, menos un término relacionado con la población de referencia usada. En el artículo anterior presenté un modelo con fricción dinámica y calculé analíticamente el indicador $\mathrm{A}+$. Aquí muestro otras propiedades de $\mathrm{A}+$, con énfasis en la interpretación física de su derivada con respecto al tiempo, $d A^{+} / d t$. Encuentro que $d A^{+} / d t$ es la media del recíproco de la escala de tiempo dinámica, ponderada por la densidad de rezagadas azules. Muestro que no es negativa, pues la densidad de las rezagadas azules no es negativa. Muestro también que si la escala de tiempo de la fricción dinámica no decrece con el radio, entonces $d A^{+} / d t$ no decrece con el tiempo, lo que hace que A+ sea una función convexa.

Key Words: blue stragglers - globular clusters: general — methods: analytical

\section{INTRODUCTION}

Alessandrini et al. (2016) recently introduced a new parameter, intended as a quantitative measurement of the dynamical age of old star clusters. This indicator, dubbed A+ for short, is based on measuring the radial distribution of massive tracers, such as blue straggler stars (Sandage 1953), which are undergoing mass segregation by virtue of being heavier than the average stars in the host cluster. The A+ indicator, in particular, involves taking the integral of the cumulative distribution of blue straggler stars

\footnotetext{
${ }^{1}$ INAF, Osservatorio Astronomico di Padova, vicolo dell'Osservatorio 5, I-35122 Padova, Italy.

${ }^{2}$ INFN, Sezione di Padova, Via Marzolo 8, I-35131 Padova,
}

as a function of log radius, minus the same for a reference population, such as horizontal branch stars. The A+ indicator is easier to measure compared to other attempts at building a dynamical clock out of the blue straggler star distribution (Ferraro et al. 2012; Hypki \& Giersz 2013; Miocchi et al. 2015; Hypki \& Giersz 2017; Pasquato et al. 2018; Sollima \& Ferraro 2019) as it does not depend on finding the exact position of the minimum of said distribution. While it was originally introduced in the context of N-body simulations, the A+ indicator was quickly shown to be able to capture the dynamical evolutionary stage of observed globular clusters, displaying a strong anticorrelation with their half-mass relaxation 
time (Lanzoni et al. 2016). Throughout their paper, Alessandrini et al. (2016) argue that the A+ indicator strictly increases with time, and indeed show that this is the case in the large set of simulations they run, spanning a wide range of initial conditions and dynamical ingredients. This holds of course except for short term fluctuations due to numerical, or possibly dynamical, noise. Monotonicity with time is a crucial property for the A+ as well as for any other indicator of star-cluster dynamical aging, which is an intrinsically irreversible process. Another property of $\mathrm{A}+$ that is apparent in the simulations run by Alessandrini et al. (2016), e.g. in their Figure 5, is that the derivative of $\mathrm{A}+$ also increases with time, i.e. that $\mathrm{A}+(\mathrm{t})$ is a convex function. In a companion paper (Pasquato 2019, referred as P19 for short in the following), I worked out an analytical formula for the (three dimensional) $\mathrm{A}+$ indicator in a simplified model of mass segregation under pure dynamical friction, and showed that it evolves monotonically as a function of time for any initial blue straggler star distribution, and any radially increasing dynamical friction timescale law. Here I build on these results to obtain a few interesting properties of the $\mathrm{A}+$ indicator. In particular I show that its derivative is a non-negative function, which is non-decreasing as a function of time if the dynamical friction timescale is non-decreasing with radius, making the $\mathrm{A}+$ indicator a convex function of time. I provide a simple formula for the value of the $\mathrm{A}+$ indicator derivative at the beginning of dynamical evolution, which directly connects it to the dynamical friction timescale. Finally, I suggest that in the late stages of dynamical evolution the A+ indicator derivative should approach a constant, making the A+ indicator asymptotically linear.

\section{CALCULATIONS}

In the context of the simplified model of evolution under dynamical friction introduced in $\mathrm{P} 19$, the $\mathrm{A}+$ indicator is calculated as

$$
\begin{aligned}
A^{+}(t)= & \int_{-\infty}^{+\infty} N\left(I^{-1}\left(I\left(e^{s}\right)+t\right), 0\right) d s- \\
& \int_{-\infty}^{+\infty} N\left(e^{s}, 0\right) d s
\end{aligned}
$$

where $N(r, t)$ is the cumulative distribution of blue straggler stars as a function of radius and time. The function $I(\cdot)$ is defined by $\mathrm{P} 19$ as

$$
I(r)=\int \frac{\tau(x) d x}{x},
$$

where $\tau(r)$ is the dynamical friction timescale as a function of radius.

\subsection{Time Derivative of the A+ Indicator}

The second term on the right hand side of equation 1 does not depend on time, so its derivative vanishes. Regarding the first term, I redefine for simplicity

$$
N_{0}(r)=N(r, 0),
$$

so

$$
\begin{aligned}
\frac{d A^{+}}{d t} & =\frac{d}{d t} \int_{-\infty}^{+\infty} N_{0}\left(I^{-1}\left(I\left(e^{s}\right)+t\right)\right) d s \\
& =\int_{-\infty}^{+\infty} \frac{d}{d t} N_{0}\left(I^{-1}\left(I\left(e^{s}\right)+t\right)\right) d s
\end{aligned}
$$

which, by using the inverse function derivative rule, becomes

$$
\frac{d A^{+}}{d t}=\int_{-\infty}^{+\infty} \frac{d N_{0}}{d r}\left(I^{-1}\left(I\left(e^{s}\right)+t\right)\right) \frac{d s}{I^{\prime}\left(I^{-1}\left(I\left(e^{s}\right)+t\right)\right)},
$$

that is

$$
\frac{d A^{+}}{d t}=\int_{-\infty}^{+\infty} \frac{d N_{0}}{d r}\left(r_{0}\left(e^{s}, t\right)\right) \frac{r_{0}\left(e^{s}, t\right)}{\tau\left(r_{0}\left(e^{s}, t\right)\right)} d s,
$$

where $r_{0}(r, t)=I^{-1}(I(r)+t)$ is the position at time $t=0$ of a star currently at radius $r$ (see P19 for a proof). Substituting back $s=\log r$, I obtain

$$
\frac{d A^{+}}{d t}=\int_{0}^{+\infty} \frac{d N_{0}}{d r}\left(r_{0}(r, t)\right) \frac{r_{0}(r, t)}{r \tau\left(r_{0}(r, t)\right)} d r ;
$$

by further substituting, for a given $t, r_{0}=r_{0}(r, t)$, I obtain

$$
\begin{aligned}
\frac{d A^{+}}{d t} & =\int_{0}^{+\infty} \frac{d N_{0}}{d r}\left(r_{0}\right) \frac{r_{0}}{r\left(r_{0}, t\right) \tau\left(r_{0}\right)} \frac{d r}{d r_{0}} d r_{0} \\
& =\int_{0}^{+\infty} \frac{d N_{0}}{d r}\left(r_{0}\right) \frac{d s}{d s_{0}} \frac{d r_{0}}{\tau\left(r_{0}\right)},
\end{aligned}
$$

where $s_{0}=\log r_{0}$. Alternatively, I can use the inverse function derivative again to obtain

$$
\frac{d A^{+}}{d t}=\int_{0}^{+\infty} \frac{d N_{0}}{d r}\left(r_{0}\right) \frac{d r_{0}}{\tau(r)} .
$$

This is a general formula for the derivative of the $\mathrm{A}+$ indicator at time $t$. It is always non-negative because the integrand is non-negative as long as the derivative of the initial cumulative distribution function is. This condition is equivalent to requesting that density is always non-negative. In other words, the monotonicity of the $\mathrm{A}+$ indicator derives from the condition that the number density of blue straggler stars is everywhere non-negative. Note how in 
equation 9 the time dependence is all enclosed within the $\tau(r)$ term (not $\tau\left(r_{0}\right)$ !) at the denominator. If $\tau(r)$ is constant I recover the result of P19 where the $\mathrm{A}+$ indicator is linear with time, as its derivative equals $1 / \tau(0)$ and its initial value is always zero by construction.

\subsection{Convexity}

The evolution of a star's radius given its initial value $r_{0}$ and the elapsed time $t$ is given by equation 3 of P19:

$$
r\left(r_{0}, t\right)=I^{-1}\left(I\left(r_{0}\right)-t\right) .
$$

For any $r_{0}, t>0$ it holds that $r\left(r_{0}, t\right)<r_{0}$ due to the monotonicity of $I(\cdot)$. Moreover, for any $t_{2}>t_{1}>0$

$$
\begin{aligned}
r\left(r_{0}, t_{2}\right) & =r\left(r_{0}, t_{1}+\left(t_{2}-t_{1}\right)\right) \\
& =I^{-1}\left(I\left(r_{0}\right)-t_{1}-\left(t_{2}-t_{1}\right)\right),
\end{aligned}
$$

and, defining $r_{1}=r\left(r_{0}, t_{1}\right)=I^{-1}\left(I\left(r_{0}\right)-t_{1}\right)$ we obtain

$$
\begin{aligned}
r\left(r_{0}, t_{2}\right) & =I^{-1}\left(I\left(r_{1}\right)-\left(t_{2}-t_{1}\right)\right) \\
& =r\left(r_{1}, t_{2}-t_{1}\right)<r_{1}=r\left(r_{0}, t_{1}\right),
\end{aligned}
$$

so for a given $r_{0}$, the function $r\left(r_{0}, t\right)$ is monotonically decreasing with $t$. Stars can only sink towards smaller radii over time. Now, if $\tau(r)$ is nondecreasing, $\tau\left(r_{1}\right) \geq \tau\left(r_{2}\right)$ for any $r_{1}>r_{2}$. Then the denominator $\tau(r)$ in equation 9 cannot increase with time because $r$ decreases as shown in equation 12, and

$$
\begin{aligned}
\left.\frac{d A^{+}}{d t}\right|_{t=t_{2}}= & \int_{0}^{+\infty} \frac{d N_{0}}{d r}\left(r_{0}\right) \frac{d r_{0}}{\tau\left(r\left(r_{0}, t_{2}\right)\right)} \geq \\
& \int_{0}^{+\infty} \frac{d N_{0}}{d r}\left(r_{0}\right) \frac{d r_{0}}{\tau\left(r\left(r_{0}, t_{1}\right)\right)} \\
= & \left.\frac{d A^{+}}{d t}\right|_{t=t_{1}},
\end{aligned}
$$

proving that $\mathrm{A}+$ is a convex function of time. This is a quite interesting result in the light of Figure 5 of Alessandrini et al. (2016) where the (two dimensional) A+ indicators calculated for different simulations appear to evolve with increasing slope for increasing times, modulo the numeric error. The physical interpretation for this result is that the evolution becomes faster as the blue straggler stars drop to smaller radii, where the dynamical friction timescale is shorter.

Equation 7 can also be used to calculate the slope of the $\mathrm{A}+$ indicator at the start of the dynamical evolution, e.g. in a simulation, by setting $t=0$.

$$
\left.\frac{d A^{+}}{d t}\right|_{0}=\int_{0}^{+\infty} \frac{d N_{0}}{d r}(r) \frac{d r}{\tau(r)} .
$$

To further carachterize this I can write

$$
\left.\frac{d A^{+}}{d t}\right|_{0}=\int_{0}^{1} \frac{d N_{0}}{\tau\left(r\left(N_{0}\right)\right)}
$$

So the derivative of the $\mathrm{A}+$ indicator at the beginning of the dynamical evolution (e.g. at the start of a simulation) is the mean of the reciprocal of the dynamical friction timescale considered as a function of the initial cumulative number distribution of blue stragglers.

\subsection{Limiting Behaviour at Large Times}

If I set $\tau_{0}=\tau(0)=\tau_{L}(0)$ and $\tau_{\infty}=\lim _{r \rightarrow \infty} \tau(r)=$ $\tau_{L}(1)$ I obtain

$$
\frac{1}{\tau_{\infty}}<\left.\frac{d A^{+}}{d t}\right|_{0}<\frac{1}{\tau_{0}}
$$

by substitution into equation 9 , as $\tau(r)$ is increasing with radius.

In general, since a constant $\tau(r)=\tau(0)$ holds for the approximately constant-density cores of globular clusters, I expect the final stages of the evolution, when most blue stragglers have sunk to the core, to have a value of the $\mathrm{A}+$ indicator's derivative that approaches the constant value $1 / \tau(0)$. As I have shown, any value attained later in evolution should be larger than previous values of the derivative. This is another point where comparison with Figure 5 of Alessandrini et al. (2016) is reassuring, as all the slopes in that figure are quite smaller than one, even though their values of the $\mathrm{A}+$ indicator are referred to the half-mass relaxation timescale, which is longer than the central dynamical friction timescale. Further caveats for a comparison are that:

- at some point during the evolution of a cluster, my simplified picture of dynamical friction will no longer represent reality, as diffusion effects will start to dominate right in the central regions, so the limiting value of $d A^{+} / d t=1 / \tau(0)$ is not necessarily reached;

- blue stragglers on very eccentric orbits already depart from my simplified picture, even at the beginning of their evolution;

- Alessandrini et al. (2016) use a projected, twodimensional version of the $\mathrm{A}+$ indicator;

- the reference stars (horizontal branch stars) they use are also evolving over time, as they are likely heavier than the average star in the simulations they run.

In subsequent papers I will address the third point, obtaining a better basis for comparison with simulations. The fourth point is addressed in the following. 


\subsection{Moving Reference Stars}

Until now I assumed that the reference stars for the $\mathrm{A}+$ indicator are fixed, i.e. that they keep the initial cumulative distribution over time. If I relax this assumption, while still assuming that the initial cumulative distribution of blue straggler and reference stars coincides, equation 1 becomes

$$
\begin{aligned}
A^{+}(t)= & \int_{-\infty}^{+\infty} N\left(I^{-1}\left(I\left(e^{s}\right)+t\right), 0\right) d s- \\
& \int_{-\infty}^{+\infty} N\left(\hat{I}^{-1}\left(\hat{I}\left(e^{s}\right)+t\right), 0\right) d s
\end{aligned}
$$

where

$$
\hat{I}(r)=\int \frac{\hat{\tau}(x) d x}{x},
$$

and $\hat{\tau}(r)$ is the dynamical friction timescale as a function of radius for the reference particles. If $\hat{\tau}(r) \gg \tau(r)$ for every $r$ then we revert back to the original situation where I can neglect the effects of dynamical friction on the reference population, while if $\hat{\tau}(r)=\tau(r)$ then the $\mathrm{A}+$ indicator will be identically zero over time. In the following I will assume that

$$
\hat{\tau}(r)=\frac{m_{B S S}}{m_{H B}} \tau(r),
$$

where $m_{B S S}$ is the mass of a blue straggler star and $m_{H B}$ the mass of a reference star. This is in line with e.g. Mapelli et al. (2004), who assume that dynamical friction scales inversely with mass. With this in mind it is easy to see that the time derivative of $\mathrm{A}+$ calculated in equation 9 becomes

$$
\frac{d A^{+}}{d t}=\int_{0}^{+\infty} \frac{d N_{0}}{d r}\left(r_{0}\right) \frac{d r_{0}}{\tau(r)}-\int_{0}^{+\infty} \frac{d N_{0}}{d r}\left(r_{0}\right) \frac{d r_{0}}{\hat{\tau}(r)}
$$

which, by substituting in equation 19, leads to the final result

$$
\frac{d A^{+}}{d t}=\left(1-\frac{m_{H B}}{m_{B S S}}\right) \int_{0}^{+\infty} \frac{d N_{0}}{d r}\left(r_{0}\right) \frac{d r_{0}}{\tau(r)},
$$

which shows that the slope of the time evolution of $\mathrm{A}+$ is merely rescaled by a constant factor. Since both the $\mathrm{A}+$ indicator referred to a static population and the one referred to a population that also suffers dynamical friction are zero at the beginning of evolution, then $\mathrm{A}+$ itself is merely rescaled by a constant factor. Thus all the results I obtained above still hold.

\section{CONCLUSIONS}

Working within the same pure dynamical friction picture I introduced in the companion paper P19, I have calculated the time derivative of the Alessandrini et al. (2016) A+ indicator and found that it is not decreasing in time if the dynamical friction timescale $\tau(r)$ is non-decreasing with radius. Thus the $\mathrm{A}+$ indicator is a convex function of time. P19 results show that in a constant dynamical friction timescale scenario the $\mathrm{A}+$ indicator is linear with time, so convexity implies that over time the $\mathrm{A}+$ indicator evolves with increasing slope becoming asymptotically linear, as the late stages of evolution take place in the uniform density cores of globular clusters, where the dynamical friction timescale becomes constant. Finally, I have shown that the choice of a reference population that is also affected by mass-segregation (as is likely the case in an observational setting) merely leads to a constant rescaling of the $\mathrm{A}+$ indicator.

This project has received funding from the European Union's Horizon 2020 research and innovation programme under the Marie Skłodowska-Curie grant agreement No. 664931. I wish to thank Dr. Pierfrancesco di Cintio, Dr. Paolo Miocchi, Dr. Alessandro Cobbe, and Dr. Stefano Pugnetti for helpful discussion on this subject.

\section{REFERENCES}

Alessandrini, E., Lanzoni, B., Ferraro, F. R., Miocchi, P., \& Vesperini, E. 2016, ApJ, 833, 252

Ferraro, F. R., Lanzoni, B., Dalessandro, E., et al. 2012, Nature, 492, 393

Hypki, A. \& Giersz, M. 2013, MNRAS, 429, 1221 . 2017, MNRAS, 471, 2537

Lanzoni, B., Ferraro, F. R., Alessandrini, E., et al. 2016, ApJ, 833, L29

Mapelli, M., Sigurdsson, S., Colpi, M., et al. 2004, ApJ, 605, L29

Miocchi, P., Pasquato, M., Lanzoni, B., et al. 2015, ApJ, 799, 44

Pasquato, M., Miocchi, P., \& Yoon, S.-J. 2018, ApJ, 867, 163

Sandage, A. R. 1953, AJ, 58, 61

Sollima, A. \& Ferraro, F. R. 2019, MNRAS, 483, 1523

M. Pasquato, INAF, Osservatorio Astronomico di Padova, vicolo dell'Osservatorio 5, I-35122 Padova, Italy (mario.pasquato@inaf.it).

M. Pasquato: INFN, Sezione di Padova, Via Marzolo 8, I-35131 Padova, Italy. 